\title{
EXCESS HEATING OF CORONA AND CHROMOSPHERE ABOVE MAGNETIC REGIONS BY NON-LINEAR ALFVEN WAVES
}

\author{
YUTAKA UCHIDA \\ Tokyo Astronomical Observatory* and High Altitude Observatory** \\ and \\ OSAMU KABURAKI \\ Dept. of Astronomy, University of Tokyo, Tokyo
}

\begin{abstract}
Excess heating of the active solar atmosphere is interpreted as the decay of MHD slow-mode waves produced in the corona through the non-linear coupling of Alfvén waves supplied from subphotospheric layers. It is stressed that the Alfvénmode waves may be very efficiently generated directly in the convection layer under the photosphere in magnetic regions, and that such magnetic regions, at the same time, provide the 'transparent windows' for Alfvén waves in regard to the Joule and frictional dissipations in the photospheric and subphotospheric layers. Though the Alfvén waves suffer considerable reflection in the chromosphere and in the transition layer, a certain fraction is propagated out to the corona. A large velocity amplitude, exceeding the local Alfvén velocity, is attained during the propagation along the magnetic tubes of force into a region of lower density and weaker magnetic field. The otherwise divergence-free velocity field in Alfvén waves, in such a case, couples with a compressional component (slow-mode waves) which again is of considerable velocity amplitude relative to the local acoustic velocity when estimated by using the formula for non-linear coupling between MHD wave modes derived by Kaburaki and Uchida (1971). Therefore, the compressional waves thus produced through the non-linear coupling of Alfvén waves are eventually thermalized to provide a heat source. The introduction of this non-linear coupling process and the subsequent thermalization of the slow-mode waves may provide a means of converting the otherwise dissipation-free Alfvén mode energy into heat in the corona. The liberated heat will readily be redistributed by conduction along the magnetic lines of force, and thus the loop-like structure of the coronal condensations (or probably also the thread-like feature in the general corona) is explained in a natural fashion. Matter density in the arches is expected to be higher than the ambient density because of the higher temperature and, consequently, greater scale height in the arches.
\end{abstract}

(Full paper is to be submitted to Solar Physics.)

\section{Reference}

Kaburaki, O. and Uchida, Y.: 1971, Publ. Astron. Soc. Japan 23, 405.

* Tokyo Astronomical Observatory, University of Tokyo, Mitaka, Tokyo.

** High Altitude Observatory, National Center for Atmospheric Research, Boulder, Colorado, is sponsored by the National Science Foundation. 